\title{
Different continuous training modalities result in distinctive effects on muscle structure, plasticity and function
}

\author{
GABRIELE PALLONE ${ }^{1}$, MATTIA PALMIERI ${ }^{1}$, IDA CARIATI $^{1}$, ROBERTO BEI $^{2}$, \\ LAURA MASUELLI ${ }^{3}$, GIOVANNA D'ARCANGELO ${ }^{1,4}$ and VIRGINIA TANCREDI ${ }^{1,4}$
}

\author{
Departments of ${ }^{1}$ Systems Medicine and ${ }^{2}$ Clinical Sciences and Translational Medicine, University of Rome Tor Vergata, \\ I-00133 Rome; ${ }^{3}$ Department of Experimental Medicine, Sapienza University of Rome, I-00161 Rome; \\ ${ }^{4}$ Centre of Space Bio-medicine, University of Rome Tor Vergata, I-00133 Rome, Italy
}

Received June 13, 2019; Accepted December 13, 2019

DOI: $10.3892 /$ br.2020.1283

\begin{abstract}
The effects of training on muscle structure are dependent on adaptive changes induced by different intensities of physical exercise. Evidence has shown that aerobic training is able to induce adaptive changes to muscle structure based on intensity. The aim of the present study was to investigate the effects of different methods of continuous aerobic training in mice using functional, morphological and biomolecular approaches. The continuous aerobic training methods used in the present study were uniform continuous training (UC), varying continuous training (VC) and progressive continuous training (PC). Mice were made to run 3 times a week for 12 weeks on a motorized RotaRod, following one of the three different training methods at different speeds. The results of the present study demonstrated that the various training methods had different effects on sarcomere length. Ultrastructural analysis demonstrated that UC training resulted in a shortening of sarcomere length, PC training resulted in an elongation of sarcomere length and $\mathrm{VC}$ training showed similar sarcomere length when compared with the control sedentary group. Additionally, succinate dehydrogenase complex flavoprotein subunit A levels in muscle tissue following VC training were higher compared with UC and PC training. Overall, the present study showed that varying exercise methods resulted in different types of muscle plasticity, and that the $\mathrm{VC}$ protocol resulted in increased coordination and strength endurance in the functional tests, in agreement with the ultrastructural and biochemical profile. These observations support the view that $\mathrm{VC}$ training may be more efficient in increasing performance and may thus form the basis of training regimens when an improvement of motor efficiency is required.
\end{abstract}

Correspondence to: Dr Giovanna D'Arcangelo, Department of Systems Medicine, University of Rome Tor Vergata, Via Montpellier 1, I-00133 Rome, Italy

E-mail: giovanna.darcangelo@uniroma2.it

Key words: varying continuous training, progressive continuous training, uniform continuous training, muscle plasticity, muscle strength

\section{Introduction}

Training programs are designed and performed to enhance both muscle strength and endurance in humans or to limit changes associated with immobilization or disease $(1,2)$. Training induces numerous physiological adaptations that facilitate exercise capacity and the ability to sustain a workload or achieve greater power output $(3,4)$. Studies have shown that training is associated with structural changes in skeletal muscle tissue, including increased muscle length, increased thickness of fibers, increased number of myofibrils and changes in the type of muscle fibers (5-9). There is also substantial evidence showing that training induces significant changes in myoglobin content, in mitochondrial function, in oxidative enzymes and substrate metabolism in skeletal muscle tissue (10-14). Training increases muscle power and size, and increased chronic aerobic exercise improves muscle size induced by resistance training $(15,16)$.

In humans, two approaches are frequently used for aerobic training, continuous training and interval training. The continuous training approach includes Uniform Continuous (UC), Varying Continuous (VC) and Progressive Continuous (PC) modalities. It has been demonstrated that the VC modality results in higher $\mathrm{VO}_{2} \max$ volumes and an improved metabolic profile in humans with metabolic syndrome (17). Previous studies have also shown that $\mathrm{VC}$ training is associated with improved myocardial function and higher HDL levels in subjects with coronary heart disease (18-20). Taken together these data suggest that $\mathrm{VC}$ may be superior to other continuous training modalities in enhancing aerobic performance. Whilst the aforementioned studies have shown that continuous training may exert specific clinical effects, their role in inducing differential changes in muscle morphology and function is not fully established.

In the present study, the structural and biochemical adaptation of skeletal muscles following various continuous training modalities in a murine mouse model was analyzed using a RotaRod system (21).

The aim of the present study was to determine whether $\mathrm{UC}, \mathrm{VC}$ and PC resulted in differential changes to muscle structure, plasticity and function thus providing insight into the biological effects and therefore possibilities for the use of each modality in training. 


\section{Materials and methods}

Experimental animals. In the present study, a total of $16 \mathrm{BALB} / \mathrm{c}$ male mice were divided into 4 groups; a control group and 3 groups of mice trained with either a UC, VC or PC protocol. Animal experiments were performed in accordance with the guidelines and regulations of the European Union Council Directive (22) and all experimental protocols were approved by the Italian Ministry of Public Health (approval no. 86/2018-PR). All efforts were made to minimize the number of animals used and their suffering. The mice started the training protocol on day $30( \pm 2)$ after birth, 6 days after weaning. The animals were well nourished and were allowed to eat until $30 \mathrm{~min}$ prior to the start of each training session. At the end of the training period, which lasted 3 months, all the mice were sacrificed on day $120( \pm 4)$ after birth. Animals were sacrificed following anesthetization with halothane as described previously (23). Muscle tissue fibers were collected from the posterior extensor, sural triceps, tibial muscle and brachial triceps.

Training system. A RotaRod 47600 (Ugo Basile Srl) was used for training animals. The RotaRod consisted of 5 cylinders covered by rubber in order to ensure optimal grip for the rodents. A total of 6 panels with a diameter of $25 \mathrm{~cm}$ divided the 5 lanes, each with a $57 \mathrm{~mm}$ width, allowing 5 animals to run simultaneously. An attached display showed types and speeds of rotation, time lapsed from the start of the training session and time from the last fall. A control panel allowed for varying angular speed within a range (2-80 RPM) and time intervals for increasing speed modes from $6 \mathrm{sec}$ to $10 \mathrm{~min}$. The diameter of the 5 cylinders was $3 \mathrm{~cm}$ with a circumference of $9.42 \mathrm{~cm}$ (1 RPM $9.42 \mathrm{~cm}$ x min).

Incremental test. To set up the correct speed of rotation (RPM) on the RotaRod for each protocol of training, an incremental test was performed before the training period with all the animals. The incremental test was useful for obtaining data regarding the mean number of falls in $1 \mathrm{~min}$ for each speed between 7-34 RPM of each untrained group.

Training schedule. Before starting the experimental training session period, all animals were exercised using a dynamometer and three trials for each animal were recorded. On the same day, an incremental test on the RotaRod was performed to allow animals to familiarize themselves with the machinery and the data were recorded. On day 2, all animals were submitted to an endurance test on the RotaRod for $30 \mathrm{~min}$ at 10 RPM; on day 3, an incremental test on the RotaRod; and from day 4 onwards, specific training was started. Animals in each group were then trained three times per week for 12 weeks.

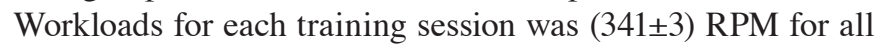
the three training regimens with a final distance for each group of $3214.266 \pm 28.278 \mathrm{~cm}$. The mean speed during UC was 64.8 and $68.4 \%$ of that recorded during VC and PC, respectively.

Fig. 1 shows the three training protocols compared in terms of speed and speed variations. During the UC training protocol a rate of $13 \mathrm{RPM}$ was set for $26 \mathrm{~min}$. During the VC protocol two speeds were used, with a higher intensity recovery period. A total of four training series were repeated within this protocol arranged into two 8 min sessions (inversion bi-pyramidal exercise). Between the two sessions a 2 min recovery rate of 10 RPM was set with a total session duration of $18 \mathrm{~min}$. Fig. 1 demonstrates how the two 8 min series were scheduled. The PC training protocol was based on a progressive 2 RPM increase rate speed from 10 RPM to 32 RPM, with 12 speed changes. The duration of the PC protocol was $18 \mathrm{~min}$.

Cross-over tests. After 10 weeks of training, all mice from each group were trained using one of the two other training schedules for one training session to determine their performance with a different protocol. Table I shows the number of falls in mice crossed-over to a different training. The number of falls was recorded after two weeks of cross-over and compared with the animals not included in the cross-over schedule.

Animal assessment. Animal weight was measured throughout the training protocols. Animals underwent a strength-endurance test using a force transducer (AdInstruments, Ltd.; cat. no. MLT050/D), where the mouse was suspended by its front legs whilst the posterior limbs were immobilized. The force transducer was connected to a computer running MLS060 Lab Chart CD 252717 (ADInstruments, Ltd.) for data recording and analysis. The time interval between the start of the test and a fall was measured, and two tests were performed for each animal prior to beginning the training protocol and following the last training session. Every test was performed three times for each animal.

Transmission electron microscopy. Muscle tissues from the left side of the animals were collected. Samples were taken from the posterior extensor, sural triceps, tibial muscle and brachial triceps. A total of 84 samples of muscle tissues were available for ultrastructure analysis. The ultrastructure analysis was performed on the extensor muscles and the samples were processed as previously described (24). Briefly, samples were fixed with $2.5 \%$ glutaraldehyde in PBS ( $\mathrm{pH} 7.4$ ), at $4^{\circ} \mathrm{C}$ for $48 \mathrm{~h}$ and then post-fixed in $1.33 \%$ osmium tetroxide for $1 \mathrm{~h}$ at $4^{\circ} \mathrm{C}$, dehydrated using a series of increasing concentrations of ethanol at $4^{\circ} \mathrm{C}$, transferred to toluene at room temperature and finally embedded in EPON 812 (Electron Microscopy Sciences) for $18 \mathrm{~h}$ at $60^{\circ} \mathrm{C}$. Thin and ultrathin sections were obtained using a Reichert ultramicrotome (Leica Microsystems $\mathrm{GmbH})$. Thin sections (1-1.5 $\mu \mathrm{m})$ were stained with toluidine blue (Sigma Aldrich; Merck KGaA) for 2 min at $70^{\circ} \mathrm{C}$ and examined under a Zeiss Axioscope (Carl Zeiss Microscopy, Inc.) light microscope at $\mathrm{x} 200$ magnification. Ultrathin sections (900 ̊) were obtained using a diamond knife (Diatome, Ltd.). Ultrathin sections were placed on copper grids, stained for $5 \mathrm{~min}$ at room temperature with uranyl acetate/lead citrate and observed using a Philips Morgagni 268D transmission electron microscope (Philips Medical Systems, Inc.) at various magnification $(\mathrm{x} 9,000, \mathrm{x} 11,000$ or $\mathrm{x} 22,000)$. The length of at least 40 different sarcomeres were evaluated in images acquired using AnalySIS ${ }^{\circledR}$ software (Philips Medical Systems, Inc.).

Western blotting. Muscle tissues from the right part of the animals were collected. Samples were taken from the posterior extensor, sural triceps and brachial triceps. Whole mounts of these muscles were taken, cut in two pieces, placed in sterile 

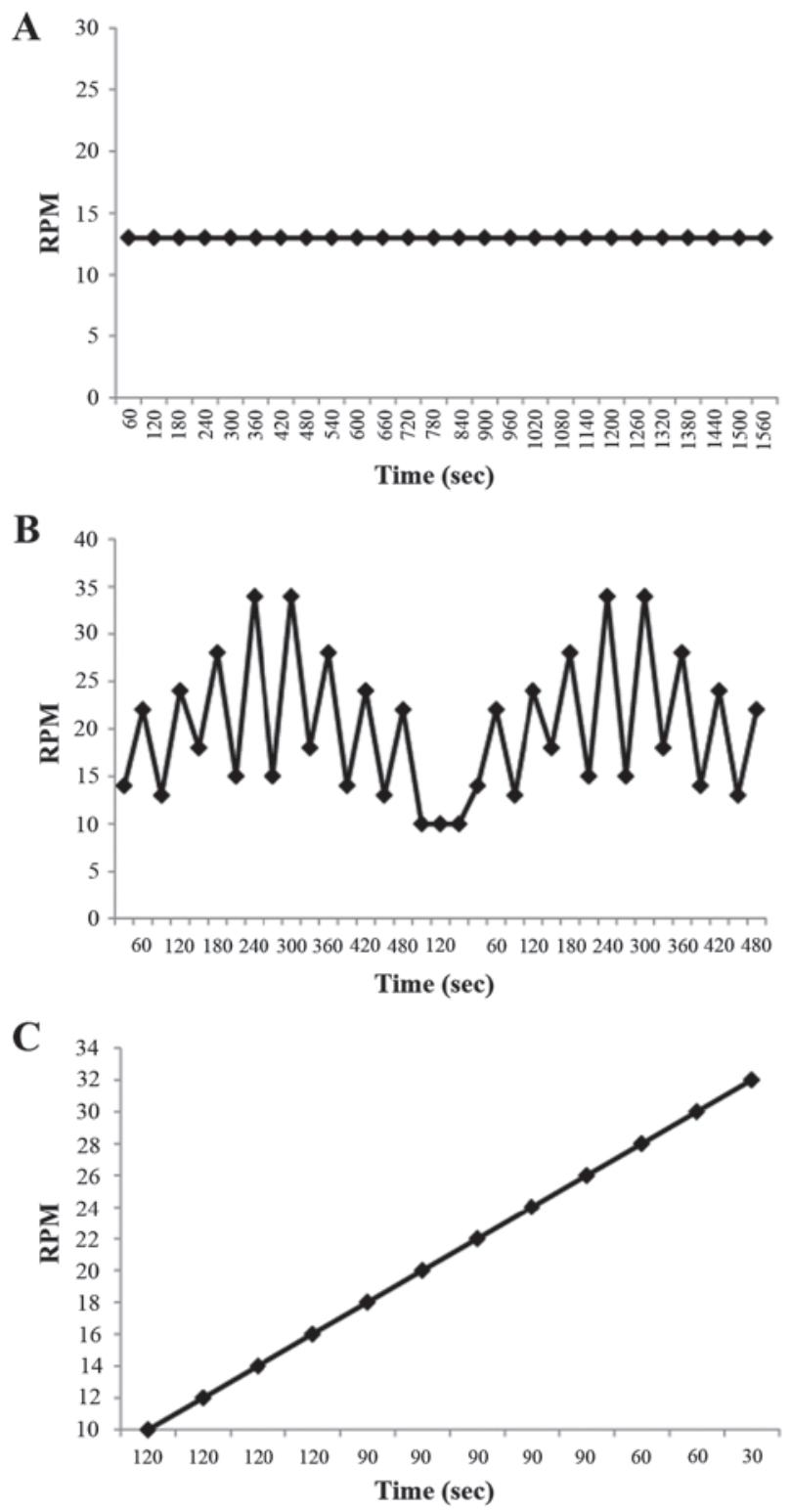

Figure 1. Comparison of three training protocols in terms of speed and speed variations. (A) The UC protocol was 13 RPM for $1,560 \mathrm{sec}(26 \mathrm{~min})$. (B) The VC protocol consisted of two series of $480 \mathrm{sec}(8 \mathrm{~min})$ of inversion bi-pyramidal exercise and an active recovery of $120 \mathrm{sec}(2 \mathrm{~min})$ at RPM between the two series, with a total training length of $18 \mathrm{~min}$. (C) The PC protocol was based on incremental speed changes with a gradually increasing intensity of exercise. The intensity increased in 2 RPM intervals from 10 RPM to $32 \mathrm{RPM}$ with 12 speed changes, for a total of $1080 \mathrm{sec}(18 \mathrm{~min})$ of training. UC, uniform continuous; VC, varying continuous; PC, progressive continuous.

plastic tubes and stored in liquid nitrogen. Two samples from each muscles were taken (6 samples from each animal), thus a total of 96 samples were available for biochemistry. Proteins were extracted from muscle tissues using a lysis buffer (10 mmol/l HEPES, 1 mmol/l EDTA, $60 \mathrm{mmol} / \mathrm{l} \mathrm{KCl}$, CA-630 $0.2 \%$ Igepal, $1 \mathrm{mmol} / 1$ sodium fluoride, $10 \mu \mathrm{g} / \mathrm{ml}$ aprotinin, $10 \mu \mathrm{g} / \mathrm{ml}$ leupeptin, $1 \mathrm{mmol} / \mathrm{l} \mathrm{DTT}, 1 \mathrm{mmol} / \mathrm{l}$ PMSF). Protein concentrations were quantified by spectrophotometry at a wavelength of $595 \mathrm{~nm}$ (PerkinElmer, Inc.). Proteins from each sample (50-100 $\mu \mathrm{g} / \mathrm{sample})$ were denatured for $5 \mathrm{~min}$ at $100^{\circ} \mathrm{C}$ in the $\beta$-mercaptoethanol, loaded on a $15 \%$ SDS gel and resolved using SDS-PAGE. Following electrophoresis, the
Table I. Number of falls in mice crossed-over to a different training protocol.

\begin{tabular}{lccccr}
\hline & \multicolumn{5}{c}{ Number of falls } \\
\cline { 2 - 6 } Groups & Mouse 1 & Mouse 2 & Mouse 3 & Mouse 4 & Mean \\
\hline UC & & & & & \\
VC crossover & 55 & 5 & 43 & 25 & 32.00 \\
PC crossover & 17 & 2 & 25 & 24 & 17.00 \\
VC & & & & & \\
UC crossover & 2 & 1 & 0 & 3 & 1.50 \\
PC crossover & 1 & 7 & 2 & 10 & 5.00 \\
PC & & & & & \\
UC crossover & 4 & 3 & 2 & 0 & 2.25 \\
VC crossover & 11 & 11 & 25 & 14 & 15.25 \\
\hline
\end{tabular}

$\mathrm{UC}$, uniform continuous; VC, varying continuous; PC, progressive continuous.

proteins were transferred to a nitrocellulose membrane, which was stained with Ponceau S $(0.2 \mathrm{~g}$ Ponceau, $1 \mathrm{ml}$ acetic acid in $\mathrm{H}_{2} \mathrm{O} 100 \mathrm{ml}$ at room temperature for $5 \mathrm{~min}$ ) which binds to the amino groups of proteins. After 2-3 washes in 1X TBS to remove the Ponceau stain, the membrane was blocked in $10 \mathrm{ml} 5 \%$ non-fat milk in TBS-0.025\% Tween (TBS-T) for $1 \mathrm{~h}$ at room temperature. Subsequently, membranes were incubated overnight at $4{ }^{\circ} \mathrm{C}$ with constant agitation with the anti-myoglobin antibody [myoglobin (A-9), sc-74525, Santa Cruz Biotechnology, Inc.] at 1:1,000 dilution in TBS-T and the anti-SDHA antibody [SDHA (B-1), sc-166909, Santa Cruz Biotechnology, Inc.] at 1:1,000 dilution in TBS-T. The following day, after $3 \times 10$ min washes with TBS-T, the membranes were incubated for $1 \mathrm{~h}$ at room temperature with a peroxidase-conjugated secondary antibody and subsequently washed with TBS-T three times. Signals were visualized using a chemiluminescence kit (Pierce; Thermo Fisher Scientific, Inc.). After developing the blots, each membrane was stripped of bound antibodies and reprobed several times. The membrane was submerged in stripping buffer $(100 \mathrm{mM}$ 2-Mercaptoehanol, 2\% SDS, $62.5 \mathrm{mM}$ Tris $\mathrm{HCl}, \mathrm{pH}$ 6.7) and incubated at $50^{\circ} \mathrm{C}$ for $30 \mathrm{~min}$ with occasional agitation. Then, the membrane was washed for $2 \times 10 \mathrm{~min}$ in TBS-T at room temperature. The membrane was blocked by immersion in 5\% non-fat dried milk in TBS-T for $1 \mathrm{~h}$ at room temperature. To ensure equal loading, membranes were incubated with a mouse-anti-human monoclonal $\beta$-actin antibody $(1: 5,000$; Sigma-Aldrich; Merck KGaA) at room temperature for $1 \mathrm{~h}$. Computer-assisted scanning densitometry (Total lab, AB.EL Science-Ware Srl) was used to analyze the intensity of the immunoreactive bands, relative to $\beta$-actin.

Statistical analysis. Data are presented as the mean \pm standard error of the mean. Comparisons between multiple groups were performed using an ANOVA, with either a post-hoc Dunnett's or Tukey's test. $\mathrm{P}<0.05$ was considered to indicate a statistically significant difference. Statistical analyses were carried out using the GraphPad Prism software version 3.02 
(GraphPad Software, Inc.). Microsoft Office Excel 5.0 software (Microsoft Corporation) was used for generation of graphs.

\section{Results}

Training increases the body weight of mice. Mice weight was measured throughout the entire experimental procedure and is shown in Fig. 2A. At 1 month of age, mean body weight prior to initiation of the training sessions was $18.5 \pm 0.6 \mathrm{~g}$ in the control group, $19.2 \pm 1.9 \mathrm{~g}$ in the UC group, $18.2 \pm 0.9 \mathrm{~g}$ in the VC group and $22 \pm 0.6 \mathrm{~g}$ in the PC group. At the age of 4 months, after training sessions were completed, mean body weight was $21 \pm 0.8 \mathrm{~g}$ in the sedentary group, $29.6 \pm 1.1 \mathrm{~g}$ in the UC group, $28 \pm 1.6 \mathrm{~g}$ in the VC group and $33 \pm 1.1 \mathrm{~g}$ in the PC group. The mean body weight increase following training was $54 \%$ in the UC group, $53 \%$ in the VC group and 50\% in the PC group, whereas in the sedentary group, an increase of only $13.5 \%$ was observed. These data show that training was associated with an increase in body weight that was statistically significant in the three training protocols compared with the control sedentary group $(\mathrm{P}<0.001)$.

Effects of different training protocols on strength. On the first and last day of training, the strength of each mouse was assessed using a dynamometer three times. The time in sec during which the animal remained suspended with their forelegs without falling was measured. Fig. 2B shows the comparison of mean suspension times before and after training. Before training, mean suspension time was $27.5 \pm 36.6 \mathrm{sec}$ in the UC group, $18.4 \pm 8.2 \mathrm{sec}$ in the VC group and $27 \pm 14.8 \mathrm{sec}$ in the PC group, and there were no significant differences between the groups. After training, mean suspension time was $23 \pm 14.1,75.3 \pm 23$ and $32.7 \pm 9.4 \mathrm{sec}$, respectively. The differences in the mean suspension time of VC compared with UC, and in VC compared with $\mathrm{PC}$ were significant $(\mathrm{P}<0.01$ and $\mathrm{P}<0.05$, respectively; Fig. $2 \mathrm{~B})$. Training significantly increased suspension time in the VC group (255\% increase), whereas in the other two groups the increase was minimal and not significant. These results suggest that the VC training protocol was associated with increased muscle efficiency and strength endurance.

Incremental test identifies four ranges of speed. To set up the correct speed of rotation, an incremental test was performed prior to the training period with all the animals used in the experiments. Data presented in Fig. $2 \mathrm{C}$ were used to determine the four ranges of speed on the RotaRod: Low intensity, $>7$ and $\leq 14$ RPM; average intensity, >14 and $\leq 20$ RPM; high intensity, $>20$ and $\leq 30$ RPM; and very high intensity, >30 RPM.

Cross-over tests show that VC training is the most efficient. During the 12 weeks of training, the number of falls for every type of protocol was evaluated. The mean number of falls during the training period were, $1.5 \pm 1.8$ in the UC group, $12.2 \pm 6.2$ in the VC group and $7.9 \pm 0.6$ in the PC group with a significantly lower number of falls in the UC group compared with both the $\mathrm{VC}$ and $\mathrm{PC}$ groups (both $\mathrm{P}<0.01$ ). These results suggest that the $\mathrm{VC}$ protocol was the most challenging training protocol. After an adaptation period of 10 weeks, mice were enrolled in a cross-over experiment to test their performance when switched to a different training protocol. When UC animals were crossed over to $\mathrm{VC}$ training, their mean number of falls was $32 \pm 21.8$ (total falls $128 ; \mathrm{P}<0.05$ compared with UC alone). When the UC mice were crossed over to PC training, the mean number of falls was $17 \pm 10.6$ (total falls 68 ), which did not differ significantly compared with the mean number of falls in the UC mice. When VC animals were crossed over to UC training, their mean number of falls was significantly decreased to $1.5 \pm 1.3$ (total falls 6 ); and after crossing over to PC training, the mean number of falls was significantly decreased to $5 \pm 4.2$ (total falls 20) (both $\mathrm{P}<0.05$ compared with VC alone). Finally, when PC animals were crossed over to UC training, their mean number of falls was $2.2 \pm 1.7$ (total falls 9), while crossing over to VC training resulted in a mean number of falls of $15.7 \pm 6.6$ (total falls 63 ) and neither differed significantly from PC alone. Comparison data of the number of falls following crossovers in the three groups are presented in Fig. 2E. Results of the crossover experiments suggested that the VC trained mice exhibited the most notable improvement in performance compared with the other two groups.

Muscle plasticity following training. To evaluate the adaptation of muscular plasticity following training with each protocol, ultrastructural analysis and measurement of sarcomere length of muscle tissues was performed (Fig. 3A-E). The length of 40 sarcomeres was quantified in each muscle sample. As shown in Fig. 3A, the ultrastructural analysis of the extensor muscle from the sedentary control mice showed a correct organization of myofibrils with normal alignment of sarcomeres (3A-a, -b, -d and -e). The mitochondria were primarily located at the periphery of the fibers and were predominantly in the condensed phase (Fig. 3A-a, -b, $-d$ and $-e)$. The sarcomere showed a regular organization of actin and myosin filaments and a well-preserved ultrastructure. The Z, I, A, H and M bands were clearly discernable as indicated in Fig. 3A-c and -f. A small dilatation of the sarcoplasmic reticulum was observed (Fig. 3A-b). The average length of sarcomeres from sedentary mice was $1.92 \pm 0.13 \mu \mathrm{m}$. The ultrastructural analysis of the extensor muscle from mice trained with the UC training showed a more compact muscle tissue with an enlargement and shortening of the sarcomeres that appeared perfectly aligned (Fig. 3B-a, -b and -c). The sarcomeres were significantly shortened compared with the sarcomeres of the untrained control mice, with an average length of $1.75 \pm 0.19 \mu \mathrm{m}(\mathrm{P}<0.0001$; Fig. 3E). Mitochondria were located in the periphery and interposed between the myofibrils (Fig. 3B-a and -b). The actin and myosin filaments appeared regularly organized with a well-preserved ultrastructure. The Z, I, A, H and M bands were clearly discernable (Fig. 3B-c). The ultrastructural analysis of the extensor muscle from mice trained with the VC protocol showed proper organization of the myofibrils with a correct alignment of the sarcomeres (Fig. 3C). Condensation of the mitochondria was slightly increased and they were located in the periphery and between the myofibrils, and a small dilatation of the sarcoplasmic reticulum was observed (Fig 3C-a and -b). The sarcomeres were well organized with proper repetition of the bands (Fig. 3C-c). The average length of sarcomeres of the $\mathrm{VC}$ trained mice was $1.96 \pm 0.12 \mu \mathrm{m}(\mathrm{P}=0.12$; Fig. $3 \mathrm{E})$. The ultrastructural analysis of the extensor muscle from the mice trained with the PC protocol (Fig. 3D) showed the organization 
A

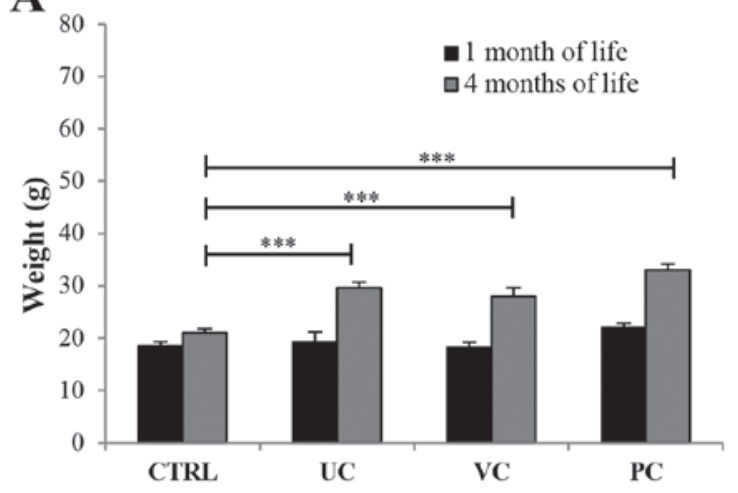

B

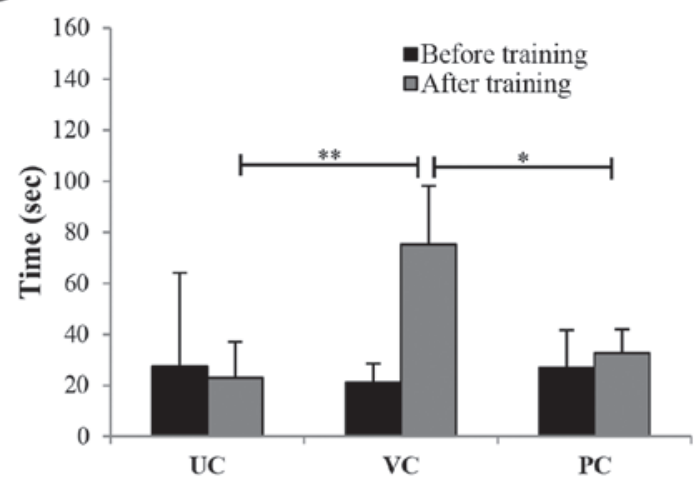

C
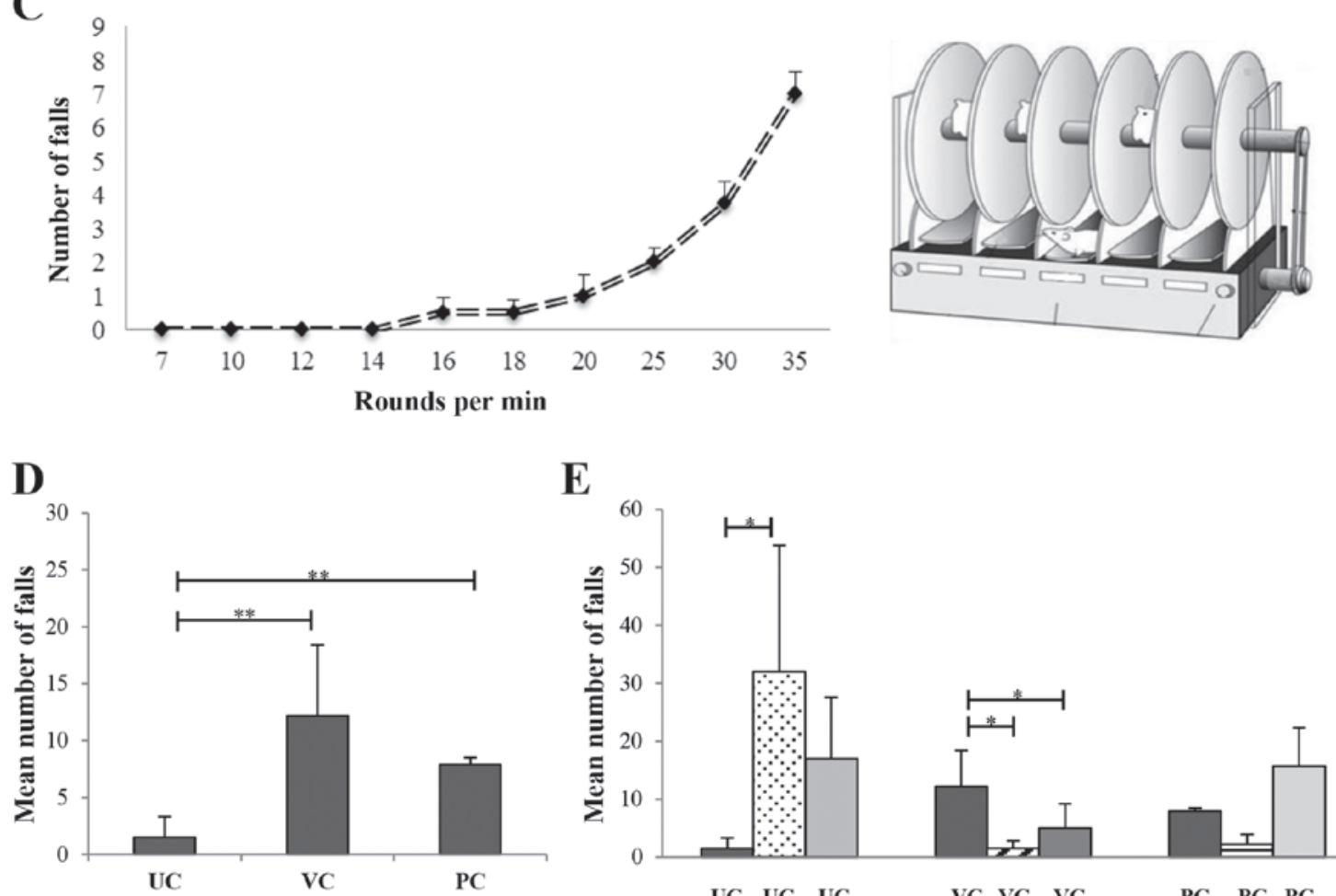

$\mathbf{E}$

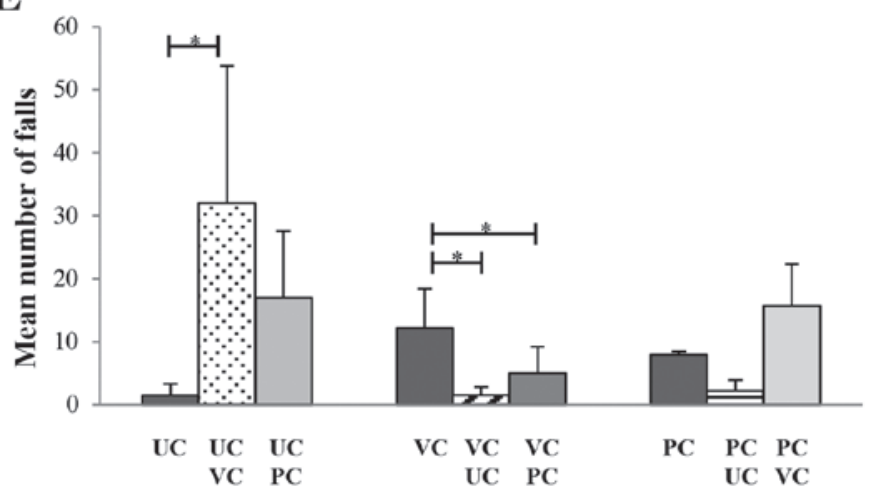

Figure 2. Effects of different training protocols on physical and performance parameters. (A) Weight variations in control and trained mice 1 and 4 months after birth. The mean body weight in the control group was $18.5 \pm 0.6 \mathrm{~g}$ after 1 month and $21 \pm 0.8 \mathrm{~g}$ after 4 months. In the mice that were to be trained, the mean body weight was $19.2 \pm 1.9 \mathrm{~g}$ in the UC group, $18.2 \pm 0.9 \mathrm{~g}$ in the VC group and $22 \pm 0.6 \mathrm{~g}$ in the PC group 1 month after birth and did not differ significantly from either the control or each other. After 4 months, the mean body weight was $29.6 \pm 1.1 \mathrm{~g}$ in the UC group, $28 \pm 1.6 \mathrm{~g}$ in the VC group and $33 \pm 1.1 \mathrm{~g}$ in the PC group, and was significantly higher in all the training groups compared with the control. (B) Mean suspension time on the dynamometer test before and after training. The mean suspension times prior to training were $27.5 \pm 36.6 \mathrm{sec}$ in the UC group, $18.4 \pm 8.2 \mathrm{sec}$ in the VC group and $27 \pm 14.8 \mathrm{sec}$ in the PC group, and there was no significant differences between the times. At the end of training, the mean suspension times were $23 \pm 14.1,75.3 \pm 23$ and $32.7 \pm 9.4 \mathrm{sec}$, in the UC, VC and PC groups, respectively. The difference in the mean suspension time of VC vs. UC and VC vs. PC was significant. (C) Incremental test performed prior to the initiation of training. There were 4 speed zones on the RotaRod: Low intensity, $>7$ and $\leq 14$ RPM; average intensity, $>14$ and $\leq 20$ RPM; high intensity, $>20$ and $\leq 30 \mathrm{RPM}$; and very high intensity, $>30$ RPM. (D) Mean number of falls during the training protocol in each of the three training groups were $1.5 \pm 1.8$ in the UC group, $12.2 \pm 6.2$ in the $\mathrm{VC}$ group and $7.9 \pm 0.6$ in the PC group. The mean number of falls was significantly higher in the $\mathrm{VC}$ and $\mathrm{PC}$ training group compared with the UC training group. (E) Mean number of falls in mice crossed-over a different protocol. The mean number of falls in the UC animals crossed over to VC training was $32 \pm 21.8$, total falls 128 ; and when crossed-over to PC training it was $17 \pm 10.6$, total falls 68 . When VC trained mice were crossed over to UC training, the mean number of falls was $1.5 \pm 1.3$, total falls 6 ; and when crossed-over to PC training the mean number of falls was $5 \pm 4.2$, total falls 20 . Mean number of falls in the PC animals crossed-over to UC training was $2.25 \pm 1.70$, total falls 9 ; and when crossed-over to VC training, the mean number of falls was $15.75 \pm 6.60$, total falls $63 .{ }^{*} \mathrm{P}<0.05,{ }^{* *} \mathrm{P}<0.01$ and ${ }^{* * * *} \mathrm{P}<0.001$. CTRL, control; $\mathrm{UC}$, uniform continuous; $\mathrm{VC}$, varying continuous; $\mathrm{PC}$, progressive continuous.

of the tissue was correct, and the presence of elongated fusiform sarcomeres was observed (Fig. 3D-a and -b). The sarcomeres were significantly elongated compared with the untrained mice, with an average length of $2.14 \pm 0.25 \mu \mathrm{m}$ $(\mathrm{P}<0.0001$; Fig. 3E). There was a slight dilatation of the sarcoplasmic reticulum and the sarcomeres showed correct repetition of the bands (Fig. 3D-c). These results suggest that exposure to different experimental training protocols induced different patterns of muscle plasticity.

Effect of training protocols on myoglobin and SDHA expression. The effects of the three training protocols on 


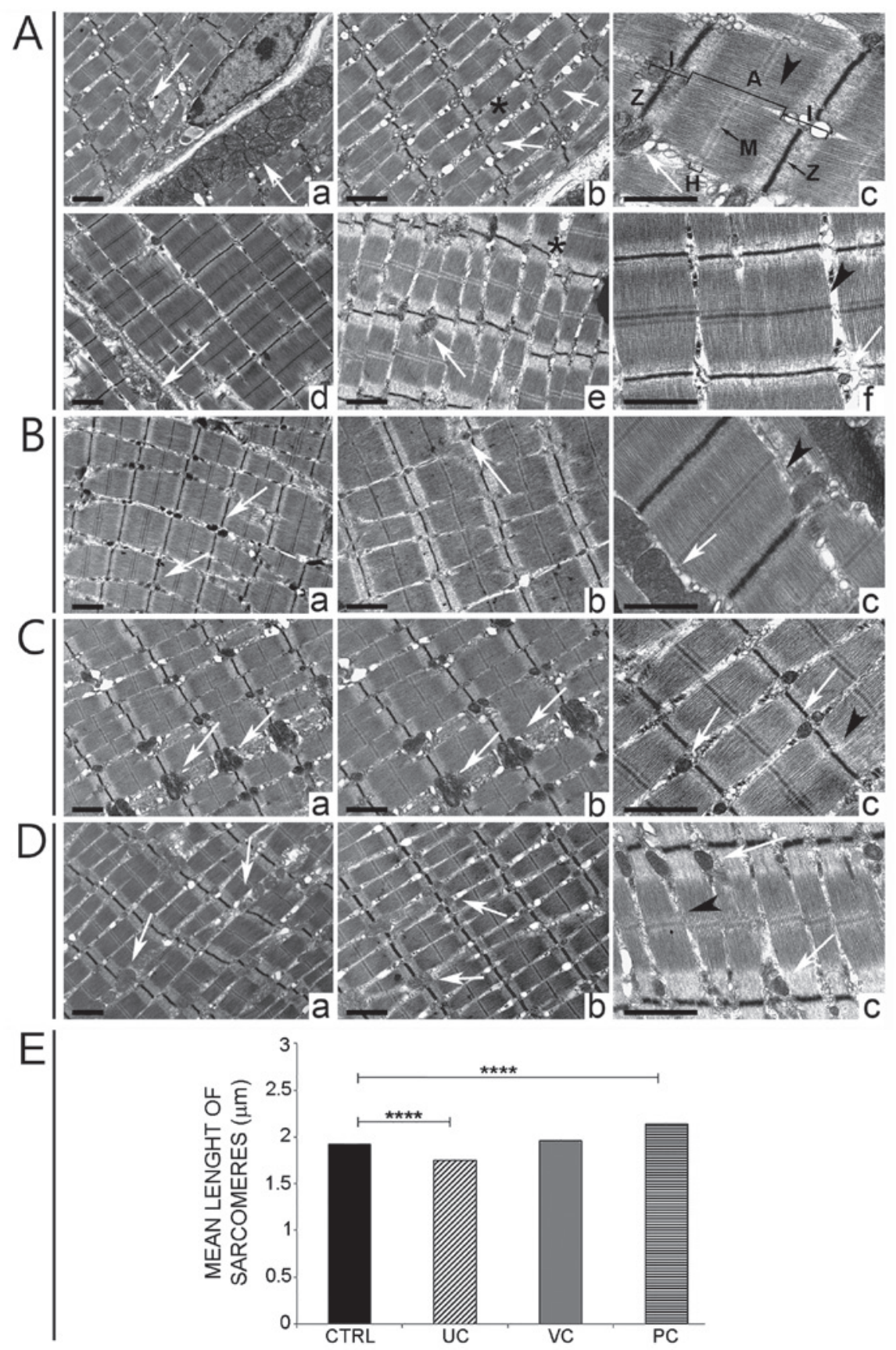

Figure 3. Muscular plasticity adaptation following training with the different protocols. (A) Ultrastructural analysis of tissue samples from extensor muscles of the control group 4 months after birth (a-f). Myofibrils were well organized, the sarcomeres were well aligned (a-f) and the mitochondria were peripherally distributed (white arrows in a-f). A small dilatation of the sarcoplasmic reticulum (in b and e) was evident. The arrowhead indicates the sarcomere between two $\mathrm{Z}$ bands and encompassing the $\mathrm{Z}$ and $\mathrm{M}$ bands (black arrows) and the I, A and $\mathrm{H}$ bands, as indicated. (B) Ultrastructural analysis of tissue samples from extensor muscles of the UC group (a-c). Several mitochondria were located between the fibers (white arrows in a-c). The arrowhead indicates the sarcomere (c). (C) Ultrastructural analysis of tissue samples from extensor muscles of the VC group (a-c). An increase in the density of mitochondria was visible amongst the fibers (white arrows in a-c). The arrowhead indicates the sarcomere (c). (D) Ultrastructural analysis of tissue samples from extensor muscles of the PC group (a-c). Elongated and fusiform sarcomeres are visible (arrowhead in c). Mitochondria were visible amongst the fibers (white arrows in a-c). Scale bar, $1 \mu \mathrm{m}$. (E) Differences in sarcomere lengths among the different training protocols. The mean lengths of sarcomeres were $1.92 \pm 0.13 \mu \mathrm{m}$ in control animals, $1.75 \pm 0.19 \mu \mathrm{m}$ in the UC group, $1.96 \pm 0.12 \mu \mathrm{m}$ in the VC group and $2.14 \pm 0.25 \mu \mathrm{m}$ in the PC group. ${ }^{* * * *} \mathrm{P}<0.0001$. CTRL, control; UC, uniform continuous; $\mathrm{VC}$, varying continuous; $\mathrm{PC}$, progressive continuous.

the expression of myoglobin and SDHA were examined and compared with the control in a total of 96 tissue samples from the posterior extensor, sural triceps and brachial triceps. As shown in Fig. 4A, the extensor muscle of the anterior limb exhibited an increase in tissue myoglobin content in all trained mice groups, but this increase was not statistically significant. The mean densitometric value of myoglobin measured in the anterior limb muscle was $1 \pm 0.5$ in the control group, $1.8 \pm 0.8$ in the UC group, $2.4 \pm 1.1$ in the VC group and $2.6 \pm 1.9$ in the $\mathrm{PC}$ group. Corresponding figures for posterior limb muscles were, $1.1 \pm 0.5,0.9 \pm 0.5,1.5 \pm 0.6$ and $1.5 \pm 0.5$, respectively (Fig. 4B), and no significant differences were 


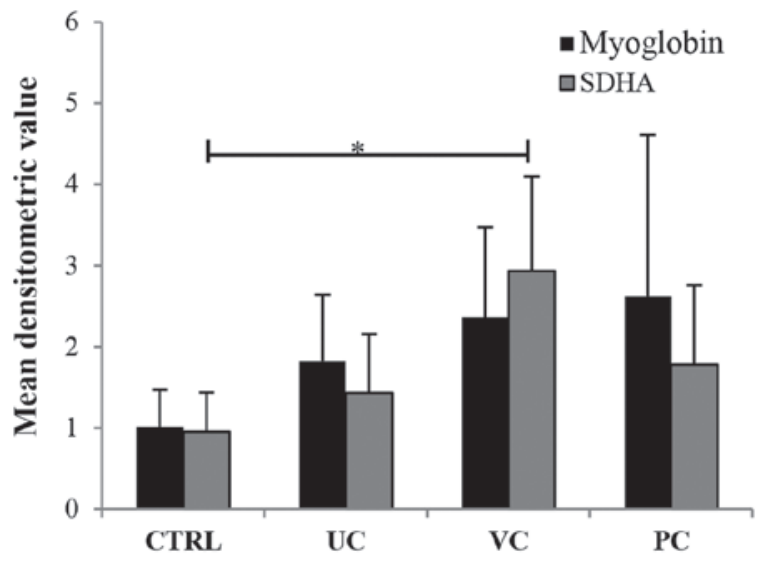

B

Posterior limb extensor muscle
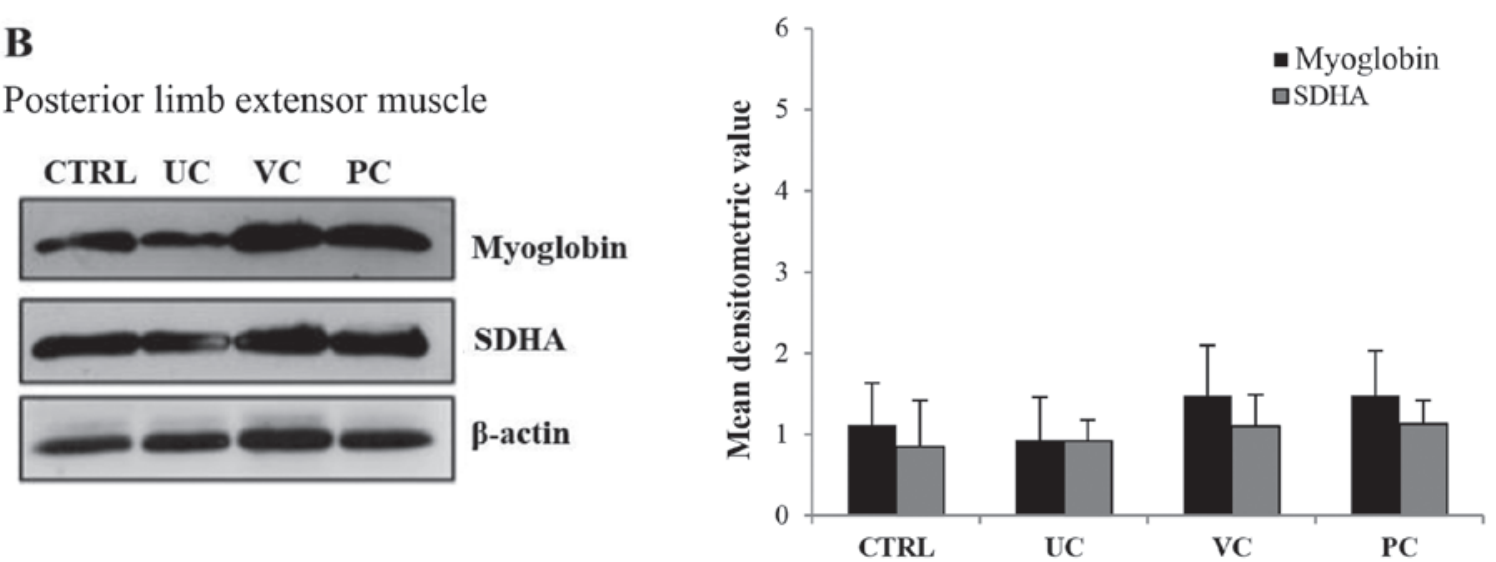

Figure 4. Effects of the three training protocols on myoglobin and SDHA expression. (A) Left, representative western blotting of myoglobin and SDHA expression in the anterior limb muscle of mice from each of the four experimental groups; right, densitometry analysis of protein expression based on western blotting. Arbitrary densitometry values for myoglobin (black bars) were: Control group, $1 \pm 0.5$; UC group, 1.8 \pm 0.8 ; VC group, 2.4 \pm 1.1 ; and PC group, $2.6 \pm 1.9$. Values for SDHA (grey bars) were: Control group, $0.9 \pm 0.5$; UC group, $1.4 \pm 0.7$; VC group, $2.9 \pm 1.2$; and PC group, $1.8 \pm 0.9$. SDHA expression in the anterior limb extensor muscles of the VC trained mice was significantly higher compared with the control mice. " $\mathrm{P}<0.05$. (B) Left, representative western blot of myoglobin and SDHA expression in the posterior limb muscles of mice from each of the four experimental groups; right, densitometry analysis of protein expression based on western blotting. Arbitrary densitometry values for myoglobin (black bars) were: Control group, $1.1 \pm 0.5$; UC group, $0.9 \pm 0.5$; VC group, $1.5 \pm 0.6$; and PC group, $1.5 \pm 0.5$; and there was no statistically significant differences between any of the groups. Values for SDHA (gray bars) were: Control group, $0.8 \pm 0.6$; UC group, $0.9 \pm 0.3$; VC group, $1.1 \pm 0.4$; and PC group, $1.1 \pm 0.3$; and there was no statistically significant differences between any of the groups. For all densitometry analysis, $\beta$-actin was used as the loading control. UC, uniform continuous; VC, varying continuous; PC, progressive continuous; SDHA, succinate dehydrogenase complex flavoprotein subunit A.

observed in the myoglobin content between sedentary control and trained mice.

Expression of SDHA was increased in the anterior limb extensor muscles of trained mice. Mean densitometric values in these samples were $0.9 \pm 0.5$ in control animals, $1.4 \pm 0.7$ in the UC group, $2.9 \pm 1.2$ in the VC group and $1.8 \pm 0.9$ in the PC group; and the increase in SDHA in the VC group was significant compared with the untrained mice $(\mathrm{P}<0.05$; Fig. 4B). No differences were observed in samples taken from the posterior limb extensor muscle (Fig. 4B), where the values were $0.8 \pm 0.6$ in the control group, $0.9 \pm 0.3$ in the UC group, $1.1 \pm 0.4$ in the VC group and $1.1 \pm 0.3$ in the PC group. These results show that only SDHA was increased significantly in muscle tissues from animals trained with VC protocols in the anterior limb.

\section{Discussion}

The present study was designed to examine the structural and biochemical adaptation of skeletal muscle to different continuous types of training modalities in a murine mouse model. The effects of continuous training modalities on muscle ultrastructure, plasticity and functional properties in mice were examined and the morphological and functional changes in experimental animals exposed to different types of continuous training were compared.

The results of the present study showed that VC-training resulted in higher efficiency gains for animals. This was particularly true in the cross-over tests and was also supported by the results of the dynamometer tests which showed that suspension times in the VC-trained animals was $255 \%$ longer than prior to training, whereas in the PC group the increase was only $21 \%$ and in the UC group, the suspension time was worse than before training, suggesting that the UC training modality may result in a decrease in strength endurance. The $\mathrm{VC}$ protocol was the most challenging protocol as demonstrated by the higher number of falls recorded in VC group during the twelve weeks of training. This increase in difficulty may be due to the 33 speed changes during each VC training session and the fact that the mean speed was slightly higher compared with the other groups. The higher challenge posed by the $\mathrm{VC}$ protocol was confirmed in the cross-over tests which showed that the number of falls of PC and UC trained mice 
was higher when crossed-over to the VC training protocol. Taken together, these data indicated that $\mathrm{VC}$ training induced higher functional efficiency as it exhibited the best results in the cross-over test and the highest improvement in the strength endurance test on the dynamometer.

While a relatively large number of studies are available on muscle hypertrophy in different clinical conditions $(25,26)$, less is known regarding the ultrastructural changes which occur in normal skeletal muscles during and after continuous training modalities. In the present study, the ultrastructural analysis of lower limb extensor muscles of mice trained with different protocols was assessed, and it showed a well-organized structure of myofibrils and sarcomeres. A small increase in the condensation of mitochondria was evident in the muscles of all trained mice, and dilatation of the sarcoplasmic reticulum was also observed, although this may have been due to the increased energy demands (27).

Analyzing the length of the sarcomeres following the different protocols showed that there was a significant decrease in sarcomere length in the UC-trained mice, and a significant elongation in the $\mathrm{PC}$-trained mice. The shortening of sarcomeres observed in the UC mice may suggest an impaired capacity to generate short bursts of high energy muscle contractions and reduced muscle tissue flexibility; whereas the elongation of the sarcomeres in the PC group may have improved flexibility and preserved the capacity to generate short bursts of high energy muscle contractions. The length of sarcomeres in the VC group was similar to that of the control group, thus suggesting that both flexibility and short burst generation capacity are preserved $(5,6,28,29)$. The variations in length of the sarcomere might be related to the motor efficiency as also suggested by the in vivo results of the cross over test.

These results appear to support the view that different patterns of muscle plasticity may evolve in response to different types of aerobic training, and that based on sarcomere length in muscle cells in the different training groups, muscle plasticity may largely depend on long term training methods. This is shown in the PC animals which exhibited a lesser enlargement of muscle tissues with little to no evidence of stress at the sub-cellular level.

In the present study, the levels of myoglobin and SDHA in the extensor muscles of both lower and front limbs in each experimental group were also assessed. Changes in myoglobin and SDHA expression was sensitive to training with the different protocols, and the relevance of these changes requires further investigation. Expression of SDHA was significantly increased only in the anterior limb muscles of the VC-trained mice. This latter observation may possibly be associated with the increased energy requirements in the VC protocol. Comparing the three training protocols directly showed that there were differences in the end results observed. The UC protocol appeared to result in less efficient coordination, strength endurance and performance on the RotaRod. The observation that sarcomere length in samples from UC-trained animals was shorter compared with the control may underlie the less efficient response in terms of strength endurance in this group. The VC protocol resulted in higher efficiency coordination, strength endurance and RotaRod test results leading to a higher performance status. The data show that mice trained with the VC protocol exhibited significantly increased levels of SDHA in the anterior limb muscle tissues compared with the control mice, suggesting enhanced aerobic metabolism (30). The PC training protocol was less efficient compared with the VC protocol in terms of coordination, strength endurance and RotaRod exercise performance, whereas the PC-trained animals performed considerably better compared with the mice trained using the UC protocol.

Whilst the data from the present study may not directly relate to humans, based on the data presented, a VC-like protocol may be proposed as a first choice approach to training when a pronounced improvement of motor efficiency is required, although additional studies in humans are required to confirm the potential beneficial effects of such a training regimen. The present study provides further evidence that varying stimulation is important for skeletal muscle adaptation, as also suggested by human-based studies on interval training (31). Additionally, studies designed to understand muscle responses to various training modalities and to explore underlying mechanisms and influencing variables may assist in optimizing exercise interventions in humans for different outcomes in different settings $(32,33)$.

\section{Acknowledgements}

The authors would like to thank Professor Andrea Modesti, Department of Clinical Sciences and Translational Medicine, Faculty of Medicine, University of Rome Tor Vergata, for the precious advice in performing ultrastructural analysis.

\section{Funding}

No funding was received.

\section{Availability of data and materials}

The datasets used and/or analyzed during the present study are available from the corresponding author on reasonable request.

\section{Authors' contributions}

GP and MP performed the animal experiments. IC performed the western blotting experiments. LM and RB performed transmission electron microscopy analysis. RB, GDA and VT wrote and edited the manuscript. GDA and VT conceived and supervised the study. All authors read and approved the final manuscript.

\section{Ethics approval and consent to participate}

Animal experiments were performed in accordance with the guidelines and regulations of the European Union Council Directive (86/609/European Economic Community) and all experimental protocols were approved by the Italian Ministry of Public Health (approval no. 86/2018-PR).

\section{Patients consent for publication}

Not applicable.

\section{Competing interests}

The authors declare that they have no competing interests. 


\section{References}

1. Costill DL, Daniels J, Evans W, Fink W, Krahenbuhl G and Saltin B: Skeletal muscle enzymes and fiber composition in male and female track athletes. J Appl Physiol 40: 149-154, 1976.

2. Harber M and Trappe S: Single muscle fiber contractile properties of young competitive distance runners. J Appl Physiol (1985) 105: 629-636, 2008.

3. Coyle EF: Integration of the physiological factors determining endurance performance ability. Exerc Sport Sci Rev 23: 25-63, 1995.

4. Hawley JA: Adaptations of skeletal muscle to prolonged, intense endurance training. Clin Exp Pharmacol Physiol 29: 218-222, 2002.

5. Fitts RH, McDonald KS and Schluter JM: The determinants of skeletal muscle force and power: Their adaptability with changes in activity pattern. J Biomech 1: 111-122, 1991.

6. Fitts RH and Widrick JJ: Muscle mechanics: Adaptations with exercise-training. Exerc Sport Sci Rev 24: 427-473, 1996.

7. Goldspink G: Malleability of the motor system: A comparative approach. J Exp Biol 115: 375-391, 1985.

8. Hegarty PV and Hooper AC: Sarcomere length and fibre diameter distributions in four different mouse skeletal muscles. J Anat 110: 249-257, 1971.

9. Widrick JJ, Stelzer JE, Shoepe TC and Garner DP: Functional properties of human muscle fibers after short-term resistance exercise training. Am J Physiol Regul Integr Comp Physiol 283: R408-R416, 2002.

10. Fitts RH: New insights on sarcoplasmic reticulum calcium regulation in muscle fatigue. J Appl Physiol (1985) 111: 345-346, 2011.

11. Groennebaek T and Vissing K: Impact of resistance training on skeletal muscle mitochondrial biogenesis, content, and function. Front Physiol 8: 713, 2017.

12. Holloszy JO and Coyle EF: Adaptations of skeletal muscle to endurance exercise and their metabolic consequences. J Appl Physiol Respir Environ Exerc Physiol 56: 831-838, 1984.

13. Hood DA: Contractile activity-induced mitochondrial biogenesis in skeletal muscle. J Appl Physiol (1985) 90: 1137-1157, 2001.

14. Schantz PG: Plasticity of human skeletal muscle with special reference to effects of physical training on enzyme levels of the NADH shuttles and phenotypic expression of slow and fast myofibrillar proteins. Acta Physiol Scand Suppl 558: 1-62, 1986.

15. Lundberg TR, Fernandez-Gonzalo R, Gustafsson T and Tesch PA: Aerobic exercise does not compromise muscle hypertrophy response to short-term resistance training. J Appl Physiol (1985) 114: 81-89, 2013.

16. Konopka AR and Harber MP: Skeletal muscle hypertrophy after aerobic exercise training. Exerc Sport Sci Rev 42: 53-61, 2014.

17. Tjønna AE, Lee SJ, Rognmo Ø, Stølen TO, Bye A, Haram PM, Loennechen JP, Al-Share QY, Skogvoll E, Slørdahl SA, et al: Aerobic interval training versus continuous moderate exercise as a treatment for the metabolic syndrome: A pilot study. Circulation 118: 346-354, 2008.

18. Wisløff U, Støylen A, Loennechen JP, Bruvold M, Rognmo Ø, Haram PM, Tjønna AE, Helgerud J, Slørdahl SA, Lee SJ, et al: Superior cardiovascular effect of aerobic interval training versus moderate continuous training in heart failure patients: A randomized study. Circulation 115: 3086-3094, 2007.
19. Alansare A, Alford K, Lee S, Church T and Jung HC: The effects of high-intensity interval training vs. Moderate-Intensity continuous training on heart rate variability in physically inactive adults. Int J Environ Res Public Health 15: E1508, 2018.

20. Gaitán JM, Eichner NZM, Gilbertson NM, Heiston EM, Weltman A and Malin SK: Two weeks of interval training enhances fat oxidation during exercise in obese adults with prediabetes. J Sports Sci Med 18: 636-644, 2019.

21. D'Arcangelo G, Triossi T, Buglione A, Melchiorri G and Tancredi V: Modulation of synaptic plasticity by short-term aerobic exercise in adult mice. Behav Brain Res 332: 59-63, 2017.

22. Louhimies S: Directive 86/609/EEC on the protection of animals used for experimental and other scientific purposes. Altern Lab Anim 2: 217-219, 2002

23. D'Arcangelo G, Grossi D, Racaniello M, Cardinale A, Zaratti A, Rufini S, Cutarelli A, Tancredi V, Merlo D and Frank C: Miglustat reverts the impairment of synaptic plasticity in a mouse model of NPC disease. Neural Plast 2016: 3830424, 2016.

24. Masuelli L, Focaccetti C, Cereda V, Lista F, Vitolo D, Trono P, Gallo P, Amici A, Monaci P, Mattei M, et al: Gene-Specific inhibition of breast carcinoma in BALB-neuT mice by active immunization with rat neu or human ErbB receptors. Int J Oncol 30: 381-392, 2007.

25. Ballesta García I, Rubio Arias JÁ, Ramos Campo DJ, Martínez González-Moro I and Carrasco Poyatos M: High-Intensity interval training dosage for heart failure and coronary artery disease cardiac rehabilitation. A systematic review and meta-analysis. Rev Esp Cardiol (Engl Ed) 72: 233-243, 2019

26. Ballesta-García I, Martínez González-Moro I, Rubio-Arias JÁ and Carrasco-Poyatos M: High-Intensity interval circuit training versus moderate-intensity continuous training on functional ability and body mass index in middle-aged and older women: A randomized controlled trial. Int J Environ Res Public Health 16: E4205, 2019.

27. Mannella CA: Structural diversity of mitochondria: Functional implications. Ann NY Acad Sci 1147: 171-179, 2008.

28. Gordon AM, Huxley AF and Julian FJ: The variation in isometric tension with sarcomere length in vertebrate muscle fibres. J Physiol 184: 170-192, 1966.

29. Herzog W and Leonard TR: Force enhancement following stretching of skeletal muscle: A new mechanism. J Exp Biol 205: 1275-1283, 2002.

30. Riis S, Christensen B, Nellemann B, Møller AB, Husted AS, Pedersen SB, Schwartz TW, Jørgensen JOL and Jessen N: Molecular adaptations in human subcutaneous adipose tissue after ten weeks of endurance exercise training in healthy males. J Appl Physiol (1985) 126: 569-577, 2019.

31. Cochran AJ, Percival ME, Tricarico S, Little JP, Cermak N, Gillen JB, Tarnopolsky MA and Gibala MJ: Intermittent and continuous high-intensity exercise training induce similar acute but different chronic muscle adaptations. Exp Physiol 99: 782-791, 2014.

32. Aisbett B, Condo D, Zacharewicz E and Lamon S: The impact of shiftwork on skeletal muscle health. Nutrients 9: E248, 2017.

33. Dobbins M, Husson H, DeCorby $K$ and LaRocca RL: School-Based physical activity programs for promoting physical activity and fitness in children and adolescents aged 6 to 18 Cochrane Database Syst Rev 2: CD007651, 2013. 